\title{
Gauge nonlocality in planar quantum-coherent systems
}

\author{
K. Moulopoulos* \\ University of Cyprus, Department of Physics, 1678 Nicosia, Cyprus
}

(October 18, 2013)

\begin{abstract}
It is shown that a system with quantum coherence can be nontrivially affected by adjacent magnetic or adjacent time-varying electric field regions, with this proximity (or remote) influence having a gauge origin. This is implicit (although overlooked) in numerous works on extended systems with inhomogeneous magnetic fields (with either conventional or Dirac materials) but is generally plagued with an apparent gauge ambiguity. The origin of this annoying feature is explained and it is shown how it can be theoretically removed, leading to macroscopic quantizations (quantized Dirac monopoles, integral quantum Hall effect, quantized magnetoelectric phenomena in topological insulators). Apart however from serving as a theoretical probe of macroscopic quantizations, there are cases (experimental conditions, clarified here) when this "gauge nonlocality" does not really suffer from any ambiguity: an apparently innocent gauge transformation corresponds to real change in physics of a companion system in higher dimensionality, that leads to physical momentum transfers to our own system. This nonlocality, together with the associated "proximity" or remote effects are then real and lead to the remarkable possibility of inducing topological phenomena from outside our system (which always remains field-free and can even reside in simply-connected space). Specific procedures are then proposed to experimentally detect such types of nonlocal effects and exploit them for novel applications. General consequences in solid state physics (such as the first violation of Bloch theorem in a field-free quantum periodic system) are pointed out, and formal analogies with certain high energy physics phenomena (axions, $\theta$-vacua and some types of Gribov ambiguities), as well as with certain largely unexplored phenomena in mechanics and in thermodynamics, are noted.
\end{abstract}

*Electronic address: cos@ucy.ac.cy 


\section{INTRODUCTION}

A universal dynamical nonlocality in two-dimensional (2D) quantum systems is reported that is of a gauge nature. This is done by demonstrating, using a gauge argument, that interesting and nontrivial physics may occur inside a 2D system with quantum coherence that is nearby magnetic or time-dependent electric field regions. First, a remote (or proximity) dynamic influence of such fields on adjacent regions in flat $2 \mathrm{D}$ space is shown to be a natural consequence of hidden Aharonov-Bohm (AB) effects (magnetic or electric) [1] combined with the absence of magnetic monopoles[2] in higher dimensionality (3D). This proximity effect is here rigorously shown to exist and to affect numerous results in the literature on extended and open arrangements with inhomogeneous magnetic fields (involving either conventional or Dirac materials), if quantum coherence parallel to the interfaces is taken into account. This has apparently not been noted in previous works - the reason possibly being that there generally remains a gauge ambiguity. It is shown that this ambiguity in the plane is actually due to the richer physics of a companion system in 3D that reduces to our $2 \mathrm{D}$ system in an appropriate limit. Under such a limiting procedure (and under certain experimental conditions) it is shown that, in fact, this "gauge nonlocality" does not really suffer from any ambiguity: an apparently innocent gauge transformation actually corresponds to real change in physics, due to nonequivalent displacements of the 3D companion relative to our 2D system (but all of them producing the same result in the proper planar limit) - and this involves physical momentum transfers to our remote system, with all the physical consequences of a genuine nonlocal effect. This nonlocality then has important applications to extended systems with adjacent time-dependent electric fields, or with adjacent adiabatically varying magnetic fields (in their intensity or in their placement in 3D space); these lead to the possibility of manufacture of interesting quantum devices that exploit the above proximity influence (i.e. of spacetime electric fluxes) to induce topological phenomena from outside the system - the simplest example being an electric flux-driven charge pumping in a modification of the well-known Laughlin's gauge argument[3] that is usually invoked for the explanation of the Integral Quantum Hall Effect (IQHE). From analysis of the 3D companion system it is made clear that the above proximity effects are not only real (can be realized experimentally), but they also give the possibility (A) of an easier experimental detection of AB effects (in a simply-connected system and 
without enclosed fluxes), and (B) of having the first example of a planar field-free periodic system (crystal) that actually violates Bloch's theorem (due to the hidden AB effect caused by the $3 \mathrm{D}$ companion). We propose specific ways through which an experimentalist can measure effects related to the above, hinting at expected behaviors in a conventional 2D solid state system (i.e. with parabolic energy spectrum), but also in graphene and topological insulator surfaces (examples of materials with low-energy linear energy spectrum). However, in a strict planar world, with complete lack of information on the 3D companion, this ambiguity may show up (actually reflecting our ignorance). It can then be theoretically removed when certain adjacent fluxes are properly quantized, which immediately suggests a natural way to eliminate the artificial effect for confined systems (closed manifolds), and we propose this (enforcement of elimination of the ambiguity, through quantization of nearby fluxes) as a criterion of proper behavior. We show that this has direct applicability even to cases when (effective) magnetic monopoles are present; the same criterion then directly leads to the quantization of certain macroscopic quantities, and this in turn leads to topological quantization of charge and response functions in a wide range of systems of current interest without further gauge considerations. Examples include the standard Dirac quantization of magnetic monopoles [2], and - by additionally invoking axion electrodynamics [4] - the integral quantization of Hall conductance in conventional 2D Quantum Hall systems, and also the "half-quantization" of the recently discussed quantized magnetoelectric phenomena in surface-gapped 3D time-reversal-symmetric topological insulators (by also demonstrating that this half-quantization basically reflects the Witten effect[5]). Finally, connections are noted with certain high-energy physics phenomena that seem to have a formal similarity ( $\theta$-vacua, and some types of Gribov ambiguities), as well as with certain areas in mechanics and in thermodynamics that are still largely unexplored; a mapping is also briefly mentioned to general spin-related phenomena, through boosts to properly moving frames, giving the possibility of studying nontrivial spin-physics by starting from purely orbital considerations, although a serious look at spin-related phenomena (including spin-orbit interactions) in this new framework is reserved for a future note. 


\section{THE SYSTEM}

Consider a flat rectangle (strip) of horizontal length $L$ in the $(x y)$-plane with periodic boundary conditions (pbc) along $L$ (in the $x$-direction), consisting of two adjacent (up and down in the $y$-direction) parts, again strips of length $L$, the one on top being empty of fields or scalar potentials (the "white" area) and the one at the bottom penetrated by a perpendicular magnetic field $B$ (the "dark" area). We start with a static and uniform $B$ (although this will be relaxed later), and we first consider a nonrelativistic quantum particle (of mass $m$ and charge $e$ ) that moves only inside the upper white area (i.e. the two areas are separated by an appropriate scalar potential wall, so that the lower dark (magnetic) area is totally inaccessible to the particle). Let us then set the origin $y=0$ at the floor of the dark area, i.e. take $(0,0)$ at the bottom left corner of the dark (magnetic) strip, the separating wall being at $y=d_{1}$, and the ceiling of the white area being at $y=d_{2}$ (which, for simplicity, we also consider to be impenetrable). The particle is therefore confined in the $y$-direction by the walls at $y=d_{1}$ and $y=d_{2}$, with periodic boundary conditions in the $x$-direction, and feels no magnetic field - the field $B$ being only in the adjacent dark "forbidden" area, that lies below the particle's white strip. The usual procedure to solve this rather trivial problem, especially for the $B=0$ case, would be to work in the gauge $\mathbf{A}=0$ everywhere inside the white region: eigenfunctions are then of the form $\Psi(x, y) \sim e^{i k_{x} x} \sin k_{y}\left(y-d_{1}\right)$ (with $k_{y}=\frac{n_{y} \pi}{d}, \quad n_{y}=1,2, \ldots$ and $d=d_{2}-d_{1}$, and with $k_{x}=\sqrt{\frac{2 m\left(\epsilon-\frac{\hbar^{2} k_{y}^{2}}{2 m}\right)}{\hbar^{2}}}$ being quantized as $\left.k_{x}=\frac{2 \pi}{L} n_{x} \quad\left(n_{x}=0, \pm 1, \pm 2, \ldots\right)\right)$, with the associated energies being therefore $\epsilon_{n_{x}, n_{y}}=\frac{\hbar^{2}}{2 m} \frac{\pi^{2} n_{y}^{2}}{d^{2}}+\frac{\hbar^{2}}{2 m}\left(\frac{2 \pi}{L}\right)^{2} n_{x}^{2}$. Let us now include the magnetic field $B$ (that is always inside the dark area only) by using a generalization of the Landau gauge (and a special case of one used by Bawin \& Burnel long ago[]]), with the origin being as noted above, namely $\mathbf{A}=-y B \hat{e}_{x}$ for $0 \leq y \leq d_{1}$, and $\mathbf{A}=-d_{1} B \hat{e}_{x} \equiv A_{0} \hat{e}_{x}$ for $d_{1} \leq y \leq d_{2}$; this gauge choice indeed satisfies that $\frac{\partial A_{y}}{\partial x}-\frac{\partial A_{x}}{\partial y}$ is $B$ inside and zero outside the dark region, and $\mathbf{A}$ is continuous at the separating wall (at $\left.y=d_{1}\right)$. Note that the particle in the white area now feels a nonzero (although uniform) vector potential, that makes wavefunctions formally pick up an extra phase factor $e^{i \frac{e}{\hbar c} \int^{x} \mathbf{A} \cdot d \mathbf{x}^{\prime}}$ (through a gauge transformation mapping trick, starting from $\mathbf{A}=0)$, so that we now have $\Psi^{(\mathbf{A})}(x, y) \sim e^{i k_{x} x} e^{i \frac{e}{\hbar c} A_{0} x} \sin \frac{n_{y} \pi}{d} y$. By then imposing the pbc in the $x$-direction, we obtain $e^{i\left(k_{x}+\frac{e}{\hbar c} A_{0}\right) L}=1$. From this, we can determine the new quantized values of $k_{x}$ and then the energy spectrum, which finally turns 
out to be

$$
\epsilon_{n_{x}, n_{y}}=\frac{\hbar^{2}}{2 m} \frac{\pi^{2} n_{y}^{2}}{d^{2}}+\frac{\hbar^{2}}{2 m}\left(\frac{2 \pi}{L}\right)^{2}\left(n_{x}-f\right)^{2},
$$

with $f=\frac{\Phi_{\text {dark }}}{\Phi_{0}}$, where $\Phi_{\text {dark }}=B d_{1} L$ is the total flux through the dark area and $\Phi_{0}=\frac{h c}{e}$ is the flux quantum. These allowed energies are actually periodic with respect to $\Phi_{\text {dark }}$ (with period $\left.\Phi_{0}\right)$ - as can be seen if, for a given $\Phi_{d a r k}$, proper shifting of the integers $n_{x}$ is made - and whenever $\Phi_{d a r k}$ happens to be an integral multiple of $\Phi_{0}$, the global spectrum is equivalent to that corresponding to the absence of the adjacent $B$ (i.e. to $\Phi_{\text {dark }}=0$, reducing to the one with $\mathbf{A}=0$ derived earlier).

The key observation is that, although the particle will never enter the dark area, its energy spectrum, and from this other measurable quantities (i.e. global electric current $J=-c \frac{\partial \epsilon}{\partial \Phi}$ ) are seen to be affected by the adjacent (forbidden) magnetic field - a type of "proximity field influence", and not the usual AB effect, since the magnetic flux is not enclosed by the region where the particle resides, but is only adjacent to it. If the origin of our coordinate system were chosen anywhere below the dark floor, the above result would seem to be originindependent. If however we chose the origin to be, e.g., at the wall separating the two areas, then this "effect" would go away. (And note that in flat space, change of origin is equivalent to a gauge transformation - see further on this later below). We observe therefore a gauge ambiguity. Hence one may well say that it cannot be a real physical effect; the theory however does predict such an "artificial" effect as a direct consequence. What is the reason behind it or what is its deeper origin? And, most importantly, is it ever possible to make any use of it experimentally? We shall answer in the affirmative, under certain conditions.

An immediate first understanding comes from the appearance of nonlocal terms in a gauge function [7, 8], see Appendix B. However, the deeper origin of this proximity field influence in flat space can be revealed through 3D folding (compatible with the pbc in the $x$-direction): we show in what follows that the above "effect" actually originates from the absence of magnetic monopoles anywhere in the embedding 3D space. Indeed, by folding in the $x$ direction to form a cylinder (by gluing the opposite vertical sides), the above gauge, now written in cylindrical coordinates, has only azimuthal component, and it is $\mathbf{A}=(0,-z B, 0)$ in the dark and $\mathbf{A}=\left(0,-d_{1} B, 0\right)$ in the white area, with $B$ always denoting the magnitude of the locally perpendicular field, that has now become the radial component of a total 
magnetic field $\mathbf{B}$ in $3 \mathrm{D}$ space. It is crucial then to note that this gauge choice leads in 3D to the additional appearance of a nonzero $B_{z}$ (component of $\mathbf{B}$ parallel to the cylinder's $z$-axis) that is inhomogeneous (generally $\varrho$ - and $z$-dependent). Indeed, straightforward calculation of the total field $\mathbf{B}$ produced by the above form of $\mathbf{A}$ (see Appendix A) leads to $B_{z}=-\frac{z B}{\varrho}$ in the dark area, and $B_{z}=-\frac{d_{1} B}{\varrho}$ in the white area. This inhomogeneous $B_{z}$ in all space is equal to exactly what is needed to give a flux (of this $B_{z}$ ) through the ceiling (say at height $z_{2}$ ) and the floor (say at height $z_{1}$ ) of any cylinder (of height $z_{2}-z_{1}$ ) that overall cancels out the flux (of $B_{\varrho}$ ) that goes through its curved cylindrical side-surface; and the flux of $B_{z}$ through the ceiling is also identical to the value of a horizontal closed integral of the corresponding $\mathbf{A}$ at height $z_{2}$ - this way directly demonstrating that the above cancellation is actually due to the standard AB effect (since the $B_{z}$-flux is enclosed by the particle's region). What we see here is simply that the total flux passing through the entire closed cylindrical surface is indeed zero (as demanded by the volume integral of $\nabla \cdot \mathbf{B}=0$ inside the whole cylinder). Hence, in the case of $z_{2}=z$ being in the white region, and $z_{1}=0$, the "proximity field influence" at height $z$ inside the white area is essentially the usual AB effect, since, as noted, a closed path in the white area encloses the flux of $B_{z}$, which is equal (up to a sign) to the "dark flux", i.e. the flux of $B$ through the entire dark strip (the contribution from the floor at $z_{1}=0$ being vanishing). This way the dark strip affects indirectly (through the companion system in $3 \mathrm{D}$ ) the adjacent white region.

Seeking an experimental realization, let us momentarily turn to a slightly different gauge, namely $\mathbf{A}=\left(0,-\frac{z B R}{\rho}, 0\right)$ in the dark area (a gauge discussed earlier[9], together with an actual realistic current distribution $\mathbf{J}$ that produces it) and $\mathbf{A}=\left(0,-\frac{d_{1} B R}{\rho}, 0\right)$ in the white area (all this being compatible with our own gauge for $\varrho \rightarrow R$ the radius of the cylinder). This gauge, produced by a $\mathbf{J} \propto-B R z \hat{e}_{\varphi}$, can be shown to lead to similar cancellations and a similar conclusion of influence of remote fields, see Appendix A. But, more importantly, in both gauges, the value of $B_{z}$ changes with the location of origin; i.e. in our first choice of gauge, it generally becomes $B_{z}=-\frac{\left(z-z_{0}\right) B}{\varrho}$ for $z$ in the dark area, and $B_{z}=-\frac{\left(d_{1}-z_{0}\right) B}{\varrho}$ for $z$ in the white area, and this can be seen as the actual source of the gauge ambiguity noted earlier; it has to do with a different flux balance (in the overall cancellation) between the ceiling, the floor and the side-surface of the considered cylinder, and this will generally give an origin-dependent flux in the ceiling - hence leading to a $z_{0}$-dependent $\mathrm{AB}$ influence at height $z$, and therefore a $y_{0}$-dependent proximity influence in the initial flat system. 
Note that, in both 1 st and 2nd choice of gauge, the point $z_{0}$ is always the point (height) where $B_{z}$ (or $\mathbf{J}$ ) vanishes (see also ref.[10], fig.3, for a related (but simpler) system) - these observations being important for our later discussion on a relevant experimental setup.

In spite of the above peculiarity however (namely, the extra appearance of a $B_{z}$ that actually has a vanishing point with a totally arbitrary location), the crucial property to note is that, when the plane is flat (i.e. in the limit $R \rightarrow \infty$ ), the above $B_{z}$ always goes to zero on the surface (for any finite $z$ ), because of its $1 / \varrho$ - dependence (whereas for the 2 nd gauge it is exactly zero on the cylinder surface because of a delta function centered on the axis, see Appendix A). Although $B_{z}$ is zero in the planar system, we see, however, that the memory of a finite "enclosed flux" in infinite 3D space remains, and it is this that actually causes the proximity field influence. It is as if the cylinder axis has moved to infinity in such a way that $B_{z}$ through the infinite space gives the same flux as for the folded system, namely $B_{z} \rightarrow 0$, but $\varrho \rightarrow \infty$ in such a way that their product is either $-y B$ (dark area) or $-d_{1} B$ (white area), which, in fact, are the correct values of $\mathbf{A}$ for our planar system, but now derived by a limiting procedure. It is also interesting to emphasize that the nonlocal term of [7, 8] for 2D static magnetic cases confirms (or, better, contains) this type of proximity influence directly in flat 2D space, without the need of any folding (or unfolding) or other limiting considerations (see Appendix B).

Regarding a connection of the above 2D ambiguity to, possibly, real physics, note that, mathematically, a gauge transformation in the planar problem (upon displacement of the origin $y_{0}$ ) is an ordinary gauge transformation (the gauge function is $\Lambda=-\Delta \Phi \frac{x}{L}$ (with $\Delta \Phi$ being the change of flux that corresponds to the change of vector potential $\Delta \mathbf{A}$ ) and is a smooth single-valued function everywhere on the plane); when however we fold into a cylinder, the corresponding $\Lambda$ turns out to be $\Lambda=-z_{0} B R \varphi$, that is basically identical to the above planar $\Lambda$, but is now multiply-valued (it has the usual discontinuity with respect to $\varphi$ appearing in all $\mathrm{AB}$ phenomena in a cylindrically symmetric configuration). Hence in $3 \mathrm{D}$ the change of origin $z_{0}$ is not unexpected to reflect real (difference in) physics (similarly to the standard $\mathrm{AB}$ effect that introduces additional physics, compared to a particle free of potentials); and, physically speaking, this has to do with the different (infinite in number) arrangements of the total magnetic field (in 3D space) that all produce the same physical arrangement on the side surface (namely the same radial field component) and therefore the same physical field-arrangement of our planar system. Note that the formal appearance 
of $\left(z-z_{0}\right)$ in $B_{z}$, actually reduces the ambiguity to one with respect to displacements of the point where $B_{z}$ vanishes. And that there is a great arbitrariness in placing the point of vanishing $B_{z}$ somewhere in 3D space, although the 2D system does not know of all this freedom - it only senses the radial field, which is always (for any of these $\mathbf{B}$-constructions) the same - in our case it is $B$ in the dark area and zero in the white. And then, any such change of the location of the vanishing point $z_{0}$ on the cylindrical surface involves relative displacements of the total $\mathbf{B}$-field in 3D space (relative to the cylinder), and this must be the source of momentum transfer to the particle. Indeed, such momentum transfer (integrated in infinite time) turns out to be equal to $q \Delta \mathbf{A} / c$ (as can be shown by following lines of reasoning similar to those of i.e. ref.[11]) and gives a physical origin to the extra phases (of $\mathrm{AB}$ type) picked up by the particle's wavefunctions upon change of $z_{0}$. The crucial element is that our original planar system, with the pbc, is an effectively compact system (since it can always be viewed as the $R \rightarrow \infty$ limit of a compact cylinder), and due to the compactification, the gauge transformations are not so innocent (they are actually singular, and hide real physics), the nontrivial effects having as origin the above noted momentum transfers. [Note also that, although in the planar system $B_{z}$ vanishes everywhere, the special point $z_{0}$ (or now $y_{0}$ ) has already been identified (as the unique point of local vanishing of $B_{z}$ in the 3D companion system) before taking the limit - something that will be of relevance in the experimental discussion later.]

To better appreciate the physics, we give in Appendix $\mathrm{C}$ a comparison to a simpler system (with an in-plane electric field $E$ in the dark area). This example demystifies somewhat this proximity effect and its gauge nature (now momentum reference levels being crucial, especially with respect to the underlying $\Phi_{0}$-periodicity, compared to the usual (and structureless) freedom associated with energy reference levels).

Effects of the above type are actually implicit in carbon nanotubes[12] (with the ambiguity now being reflected in a quasiperiodicity parameter), and also have immediate applicability to planar graphene (with no curvature) [13], although the above ambiguity has not to our knowledge been discussed (or exploited) - see however Appendix I for our own suggestions on what to expect in such proximity measurements in graphene and topological insulator surfaces. 


\section{GENERALIZATIONS}

Let us briefly point out some consequences on previous works, and discuss certain important generalizations, as well as issues of experimental relevance (on how i.e. these proximity influences could be detected in the laboratory): (i) The above types of effects also appear in connection with the concept of "effective scalar potential" that has been extensively used in previous works (both on conventional systems[14] and on Dirac materials[15, 16]) and in cases that the field is accessible to the particle (although this is not the focus of the present work - the case of "forbidden" fields making our proximity effect more "striking" (or physically unexpected), see Appendix D). The above noted gauge ambiguity applied here shows up as a gauge-dependence of the effective scalar potential (that seems to have escaped notice in previous works, amounting to a large number of articles by different groups), and it seems to affect even the qualitative form of this potential in the white area, bringing about important changes in measurable quantities in either conventional or Dirac systems (briefly discussed in Appendix D). (ii) The above folding procedure of our dark-and-white system actually generalizes Laughlin's gauge argument on a cylinder[3], where, however, the presence of the above $B_{z}$ is, to our knowledge, rarely discussed. The addition of our white strip on the surface of the usual Laughlin cylinder gives nontrivial consequences whenever the outside magnetic flux is not quantized (see Appendix E, on effective pumping and IQHE conditions induced from the outside). (iii) One should note that all the phenomena predicted here should be observable, independent of our (or any other) analysis of the $z_{0^{-}}$ ambiguity. One can give $z_{0}$ an absolute meaning (for a particular cylindrical system in the laboratory): it is the point in the $3 \mathrm{D}$ folded system at which the $z$-component $B_{z}$ of the total 3D magnetic field $\mathbf{B}$ (or its source, the current density $\mathbf{J}$ ) vanishes. We can therefore

determine this point $z_{0}$ in our $3 \mathrm{D}$ setup (see i.e. in fig.3 of ref.[10] the point where the magnetic lines are curved in opposite directions), and then be careful to place our system of interest (i.e. a strip with no field, exhibitting quantum coherence parallel to the interface with the dark magnetic region) in a manner so that its basis (namely the interface itself) is displaced (by a small distance $d_{1}$ ) with respect to $z_{0}$. Then, if this distance $d_{1}$ is such that the outside magnetic flux is not quantized, then the above effects (a proximity influence of this flux) should be present and measurable. (If they are not ever found, then something is wrong with standard quantum theory and/or (classical) electromagnetism). And, as shown 
earlier by a limiting procedure, these proximity influences must survive even after the system becomes flat. However, a question arises about cases when we start with a strictly flat system, with no knowledge of the location of the $B_{z}$-vanishing point of a corresponding $3 \mathrm{D}$ companion. For such cases, we will argue that we have two options to consider: for the 1st, see Appendix F (where it is shown that a possibility still remains to have a nonlocal effect with no ambiguity), and the 2 nd is the case of actually having the $y_{0}$-ambiguity, which is now physically unacceptable, and then our criterion of proper behavior (noted earlier) must be enforced. This enforcement of elimination of the ambiguity then seems to lead to (a) topological physics (manifested as quantization of certain quantities, such as magnetic charge and response functions), as well as to (b) connections and formal analogies with other physics areas. Indeed, (a) recall that, in all the above, essential use was made of the nonexistence of magnetic monopoles in $3 \mathrm{D}$ (the $\nabla \cdot \mathbf{B}=0$ law). But what if we had assumed that magnetic monopoles exist? Our simplest finding on this (see Appendix G) is that imposition of our criterion of proper behavior (forced elimination of the $z_{0}$-ambiguity) leads to quantization of fluxes external to the "white" system, so that, in the limit that our white system shrinks to zero, the nonlocal term of [7, 8] can serve as a probe of quantization of the flux through the magnetic region; and the enforced quantization of the nonlocal term leads, in turn, to the quantization of magnetic charge according to the Dirac condition[2], and more generally, to the quantization of other macroscopic quantities, that are related to quantized magnetoelectric effects in an axion electrodynamical consideration[4] (see Appendix H). In particular, our criterion seems to nicely complement the recent proof of the $2 \pi$-periodicity of the axionic action[17] by providing a justification of the quantizations of certain separate 2D fluxes (one in space and one in spacetime) that are crucial in the proof, justification that is not given in ref.[17] (see Appendix $\mathrm{H}$ for details). (b) Apart from the above, there are much wider implications (mainly physical), but also relationships with other physics areas that one can see formal analogies with (see Appendix J on axions, $\theta$-vacuum sectors [18, 19], Gribov copies[20], but also connections with certain open problems in mechanics[21] and in thermodynamics[22]), that certainly necessitate further investigation of an interdisciplinary character. 


\section{DISCUSSION}

Even however the simplest outcome of the present theoretical work - that it is in principle possible to have effects without fields, in the simply-connected plane, that originate from outside of our system and that affect its physical properties - is remarkable, and if true, extremely important in experimental work on fundamental physics as well as in practical applications. First, the most obvious use is for an easier experimental detection of AB effects: these can be indirectly measured in a simply-connected system and without enclosed and confined fluxes in the laboratory - hence with considerably lesser problems of leakage of magnetic lines, compared to the enclosed confined configurations typically used. Then, the already noted possibility of violation of Bloch theorem (especially if our "white" (no-field) system is periodic along the interface direction) is worth emphasizing. The violation is due to the presence (on the system) of the extra vector potential (from proximity with the outside $B$-field), hence due to the hidden AB effect caused by the 3D companion system, and it leads to AB-type of modifications of translation operators etc. that are used in the standard proof of the Bloch theorem. Note that these modifications are not the same as the well-known modifications of Bloch theorem in an IQHE system (such as the ones studied i.e. in [23]) with the particle being inside a field - in our case we always have $B=0$ on the particle. We therefore eventually expect modifications in the form of wavefunctions (essentially of an ABtype), and these will now be different from the standard Bloch forms; in such a case, one can first gauge away the "proximity-induced A", with the consequence of the extra appearance in the boundary conditions of a crystal momentum (parallel to the strip) that is essentially the kinematic momentum. And then, by adiabatically changing the special point $y_{0}$ in a direction transverse to the strip by a "cycle" (meaning that the corresponding change of flux is equal to $\Phi_{0}$, see Appendix $\mathrm{E}$ ), we can have the crystal momentum moving from one edge to the other of the (parallel) Brillouin zone, and hence induce new effects (or transitions, in a one-electron picture - not to mention the possible novel effects in the presence of electronelectron interactions, such as formation of composite fermions at zero magnetic field [24]). It is also interesting, and potentially useful experimentally, that, in cases when both electrons and holes are considered, the Berry phase picked up during such a cycle seems to contain not only an AB part (as derived by Berry in the transported rigid box around an AB flux[25]), but also a term directly related to the electric current, similarly to what happens in an 
AB ring [26, 27]. Finally, a periodic (or even quasiperiodic, i.e. Fibonacci) arrangement of magnetic strips (on a cylinder, or in the plane with pbc parallel to the strips), each one containing a rational flux $f \Phi_{0}$ (with $f=\frac{p}{q}, p, q$ integers), would be an interesting system to consider, with new (in)commensurability effects expected (not of the Hofstadter type[28] where we have a nonzero $B$-field), that will be a result of the interplay between the gauge nonlocality of this work and the (quasi)periodicity of the structure - behavioral patterns that will be possibly useful for novel applications in intelligent devices.

\section{CONCLUSIONS}

Regarding all the above, it is for the experiment to give the verdict, but it is fair to say that we have provided in this work strong theoretical evidence (in fact a rigorous proof) that the physics of a system can partly be dictated not only by local physical laws but also by nonlocal influences (from remote regions in spacetime) with a gauge character. We reemphasize that, because of this, it is in principle possible to have effects without fields, in the simply-connected plane, that originate from outside of our system and that affect its physical properties something remarkable, and important at least for novel applications. And although we have focused on orbital physics, there are well-defined steps (through boosts to properly moving frames) that lead to spin-physics as well (see Appendix J, on how a hidden Aharonov-Casher effect [29] is also expected to be involved). Of course one can simply take spin into account in all the above problems in a direct formal manner, or in a similar fashion to calculations that have been carried out in graphene or in other Dirac systems, when these are in $A B$ configurations (see i.e. Appendix I) - although a generalization of the U(1) gauge character of the nonlocal effects proposed here to cases with a spin-orbit coupling (now with an $\mathrm{SU}(2)$ character) would have an additional importance for modern applications and, as already noted, deserves a separate note. This arguably demonstrates that, if the above proximity effects turn out to be real, the experimental and application possibilities of exploiting them, as well as their generalizations, seem to be almost endless. 


\section{APPENDICES}

\section{A. Cylindrical geometry and flux-cancellations}

Recall that $(\nabla \times \mathbf{A})_{\varrho}=\frac{1}{\varrho}\left(\frac{\partial A_{z}}{\partial \varphi}-\frac{\partial\left(\varrho A_{\varphi}\right)}{\partial z}\right),(\nabla \times \mathbf{A})_{\varphi}=\left(\frac{\partial A_{\varrho}}{\partial z}-\frac{\partial A_{z}}{\partial \varrho}\right)$ and $(\nabla \times \mathbf{A})_{z}=$ $\frac{1}{\varrho}\left(\frac{\partial\left(\varrho A_{\varphi}\right)}{\partial \varrho}-\frac{\partial A_{\varrho}}{\partial \varphi}\right)$. For our gauge $\mathbf{A}=(0,-z B, 0)$ in the dark area and $\mathbf{A}=\left(0,-d_{1} B, 0\right)$ in the white area, these lead to: $B_{\varrho}=\frac{1}{\varrho}\left(-\frac{\partial}{\partial z}(-\varrho z B)\right)=B$ in the dark area, and $B_{\varrho}=$ $\frac{1}{\varrho}\left(-\frac{\partial}{\partial z}\left(-\varrho d_{1} B\right)\right)=0$ in the white area as required; we also obtain $B_{\varphi}=0$ in both areas,

and finally $B_{z}=\frac{1}{\varrho}\left(\frac{\partial}{\partial \varrho}(-\varrho z B)\right)=-\frac{z B}{\varrho}$ in the dark area, and $B_{z}=\frac{1}{\varrho}\left(\frac{\partial}{\partial \varrho}\left(-\varrho d_{1} B\right)\right)=-\frac{d_{1} B}{\varrho}$ in the white area. To make the cancellations of the text easily visible, take the special choice $z_{1}=0$ (the floor of the cylinder being at the origin (at the floor of the dark strip)) and for $z_{2}=z$ (the ceiling of the cylinder, lying either (a) inside the dark or (b) inside the white area); the curved side-surface then consists of either (a) just a lower part of the dark strip or (b) the entire dark area (a full curved strip, going around the axis and always lying on the curved cylindrical surface) together with a lower part of the white folded strip. Then indeed, the flux of $B_{z}$ through the ceiling is $\iint B_{z} \varrho d \varrho d \varphi=-z B 2 \pi R$ if $z$ is inside the dark area, or $-d_{1} B 2 \pi R$ (hence a constant) if $z$ lies inside the white area; and we see that, in either case, it indeed cancels out the radial $B$-flux (which is $z B 2 \pi R$ in the dark area and the constant $d_{1} B 2 \pi R$ in the white area, either of which can de determined by use of the proper $B_{\varrho}$ as given above); and the flux of $B_{z}$ through the ceiling is also identical to the value of a closed integral of the corresponding $\mathbf{A}$ around the cylinder (which is $\int A_{\varphi} R d \varphi=-z B 2 \pi R$ or $\left.-d_{1} B 2 \pi R\right)$ as expected, this way clearly demonstrating that the above cancellation is actually due to the standard AB effect (due to the $B_{z}$-flux that is enclosed by the particle's region).

In the 2nd gauge discussed in the main text, namely $\mathbf{A}=\left(0,-\frac{z B R}{\rho}, 0\right)$ in the dark area and $\mathbf{A}=\left(0,-\frac{d_{1} B R}{\rho}, 0\right)$ in the white area, from application of the above we see that the radial magnetic field is not a constant $B$ in all space (for $z \leq d_{1}$ ) as before, but it is now $B_{\varrho}=\frac{B R}{\varrho}$; we see therefore that we also have flux-cancellations that occur radially, namely through any internal cylindrical and any external cylindrical surface as expected. It also naively seems that $B_{z}$ goes away (since $\varrho A_{\varphi}$ is now independent of $\varrho$ ), which however is only true for $\varrho \neq 0$; if $\varrho=0$ is included, $B_{z}$ actually becomes proportional to a 2 -dimensional delta function at $\varrho=0$ : it is well-known in the AB literature (and is a crucial result in the 
Chern-Simons transformation in many-body physics that leads to composite fermions) that $\nabla \times \frac{1}{\varrho} \hat{e}_{\varphi}=2 \pi \delta^{(2)}(\varrho) \hat{e}_{z}$, so that the curl of the above $\mathbf{A}$ has (in the dark area) a $z$-component equal to $-2 \pi R z B \delta^{(2)}(\varrho)$, giving again a return flux along $z$-axis for this choice as well, that will cause similar cancellations as above (i.e. fluxes in the $z$ and radial directions will again cancel out). So, overall, we have the same physical interpretation as in the 1st gauge.

\section{B. Nonlocal Terms}

An immediate first understanding of the proximity influence of adjacent fields (that will also be practically useful later (as a probe, or detector of quantizations)) comes from a recent theory [7, 8], that leads to cases where the "gauge function" $\Lambda$ (defined by $\mathbf{A}=\nabla \Lambda$ at the point of observation $(x, y))$ does not only contain the standard integrals over potentials, but can also contain nonlocal terms of remote fields (this occurring whenever the paths of integrals pass through these fields - the point of observation being however always outside them). This is exactly what we are witnessing in the first example of the main text (and in the simplest possible static magnetic case). Indeed, if we use the 2nd solution of ref.[7] for $\Lambda$ (see eq.(9)), where we have vanishing integrals over paths, then we find a nonzero nonlocal term, namely $\Lambda \backsim \int_{y_{0}}^{y} \int_{x_{0}}^{x} \mathbf{B}\left(x^{\prime}, y^{\prime}\right) d x^{\prime} d y^{\prime}$ that must be independent of $y$, as required by an attached condition (constraint) to this particular solution (see condition in eq.(9) of ref.[7]). This 2nd solution is, for the particular case of our horizontal strip, indeed independent of $y$ [if we move the point of observation $(x, y)$ up and down, the flux enclosed inside the "observation rectangle" does not change], hence it is acceptable; it actually turns out to be $-\Phi_{\text {dark }} \frac{x}{L}$, and this also yields the same result as in the main text (namely $\Lambda=-\Phi_{\text {dark }} \frac{x}{L}=A_{0} x$, and the rest can be worked out as before, leading again to eq.(1) of the main text). As seen from the above, the origin-dependence is built in the form of the nonlocal terms (so they are expected to offer a natural language to describe these proximity influences and the possible gauge ambiguities - see later sections on how quantizations emerge from essentially this). Also note that, in the main text, the nonlocal terms were shown to contain (or to have knowledge of) the hidden AB influences in higher spatial dimensionality, without the need of folding or unfolding or other limiting considerations (see main text).

The exact results of the theory of nonlocal terms of refs. [7, 8] have recently been derived starting from a rather surprising advance in elementary calculus[30] - based on local ex- 
pansions in 2D around the observation point, combined with further geometric reasoning in properly applying Stokes' theorem - and apparently they have been overlooked in the physical but also in the mathematical literature (as generalized solutions of $\nabla \Lambda=\mathbf{A}$, on the simple-connected plane, when $\boldsymbol{\Lambda}$ is not defined as a decent function everywhere on the plane (i.e. due to the presence of remote fields)); it has also been proven[31] that these nonlocal forms - highly nonlinear in the potentials - are also Lorentz invariant, nicely generalizing therefore the usual 4-vector form of the standard electromagnetic Lagrangian (in its interaction with matter fields) that is only linear in the potentials.

In the discussion in Appendix E later, on possible "proximity devices", it will be noted that one can use other more sophisticated types of procedures (of inducing topological phenomena from outside the system) based on nonlocal terms in [7, 8] that involve $t$-dependent electric fields and electric scalar potentials. In this context it should be reminded that such t-dependent nonlocal terms were shown in ref. [7, 8] (through examples with involvement of electric fields) to indirectly protect relativistic causality by leading to cancellations of causality-violating terms (that seem to be silently accepted in current theories). Hence one may expect in the remote field-influence on such devices some type of "causal indeterminism" (with causality "hidden" in the phases of the quantum wavefunctions (by exploiting the Lorentz invariance of the time-dependent nonlocal terms, that we noted above)), and this actually occurring without the involvement of uncertainty principle (that currently seems to be considered absolutely necessary, to protect from causality-violation[32]). Summarizing, it seems that, generally speaking, in a number of different ways one can induce conditions of, at least topological (quantized) pumping of some quantity, resulting from manipulations from outside of our system, and in ways that are expected to respect relativistic causality. [See also how the nonlocal term-solutions can be related to irreversibility in thermodynamics and to recent open problems in mechanics, in Appendix J.]

\section{Comparison with a simpler problem}

To better appreciate the physics of our first example in the main text, let us make a physical comparison with a different (and simpler, or more familiar) system: if, instead of a $B$ across the dark region, we had chosen an in-plane electric field $\mathbf{E}$ (static and homogeneous, with i.e. direction pointing downwards), then there would still be a gauge (or 
origin-dependent) ambiguity (now for the scalar potential $V$ ), attributed to a freedom of choice of energy-reference level. Indeed, inside the dark region we would now have a linear scalar potential increasing upwards, so that, if we chose again to set the zero of potential at our origin (hence to ground the bottom of the dark region), then the bottom of the white area (and from that point upwards, the entire white area) would have a nonzero constant and uniform scalar potential (equal to $+E d_{1}$ ), since the whole white area, where our particle resides, is an equipotential region - like a region outside the positive plate of an ideal plane capacitor whose negative plate is grounded. This constant potential (and through this the outside (adjacent) field $E$ ) will contribute additively to the particle energies; and although this additivity is physically obvious, one can also formally see it through the use of gauge transformations in full generality, see below - note in particular that, if the electric field did not last for ever (i.e. if it were generally $t$-dependent), physics would be affected differently. And if we now displace the origin (hence the zero-level of scalar potential), the value of potential inside the white area - and hence the energies of the particle - will change accordingly (the "priviledged case" being to ground the ceiling (rather than the floor) of the dark area, so that we have no effect at all). In any case, it seems that even in this trivial problem, we can have an influence of an outside (adjacent) field, and also an ambiguity of this influence with respect to the placement of some origin: there is an infinite freedom of choice of this origin that gives (additively) different energies to our particle. But, again, such changes (of gauge) would be considered as innocent (and natural), namely as mere changes of energy-reference level. In our case of outside $B$ and the involvement of vector potentials (as in the examples of the main text), we actually witness a similar ambiguity (it is again a freedom of choice of a reference level), but it now concerns momenta (rather than energies). And this, we claim, introduces useful and interesting physics, basically because of the manner in which the vector potential couples to the Hamiltonian (the minimal coupling) and also because of the structure underlying the freedom of choice of momentum reference levels (namely, the periodicity, with period $\Phi_{0}$ ) - a property that the (simply additive) energies do not have.

Finally, concerning a brief argument in order to see the formal difference between the two cases compared above (one with static magnetic and the other with static electric field in the dark region), note that the nonlocal term in general time-dependent cases of ref.[7, 8] for an arbitrary electric field $\mathbf{E}$ has the form $\Lambda(y, t)=-c \int_{t_{0}}^{t} d t^{\prime} \int_{y_{0}}^{y} d y^{\prime} E\left(y^{\prime}, t^{\prime}\right)$ 
which, in our case of homogeneous and static $\mathbf{E}=E \hat{e}_{y}$ and with grounded the basis of the dark strip, is simply $-c V$, with $V$ the value of the scalar potential on the ceiling (and inside the entire white area, being $+E d_{1}$ ) - we see therefore that the outside (and inaccessible) electric field contributes to the white area even in this trivial situation. Indeed, the above $\Lambda$ leads to time-dependent wavefunctions in the $y$-direction that will now be of the form $\Psi(y, t) \sim e^{i \frac{q V}{\hbar}\left(t-t_{0}\right)} e^{-i \frac{\epsilon}{\hbar}\left(t-t_{0}\right)} \psi(y)$, which, after separation of the $t$-variable will lead to a static Schrödinger equation for $\psi(y)$ that will now have as a parameter the combination $(\epsilon-q V)$ rather than just the energy parameter $\epsilon$, leading at the end to energies of our particle that are $\epsilon(V)=\epsilon(V=0)+q E d_{1}$, namely just an additive contribution to the energies (due to the scalar potential on our system, or, note, due to the outside field) as physically expected. (Note again that, if the electric field were $t$-dependent, things would not be so trivial). However, as already noted, our case of an outside magnetic field is much more interesting: energies at the end are now affected not additively, but indirectly through the changes of momenta (because of the minimal coupling of the vector potential in the hamiltonian) and with the $\Phi_{0}$-periodicity being essential, as discussed in the main text.

\section{Accessible fields}

Here is a brief discussion on proximity cases with fields that are accessible to the particle (which are of course easier to achieve experimentally - although such cases of particles entering the adjacent fields may take away the "mystery" of the proximity field-influence, and is not quite the focus of the present work). However, it is important to point out that systems with magnetic strips or barriers have been often discussed in the literature (for accessible fields) by matching methods, through the use of the effective scalar potential noted in the main text, and this has been done for both conventional systems and Dirac materials. First, in a setup such as the text's orthogonal strip with a conventional parabolic hamiltonian, the effective scalar potential (which is $k_{x}$-dependent) contains $A_{x}(y)$, and this makes it gauge-dependent, something that also seems to have escaped notice. It is important to emphasize again that the changes of gauge do not cause changes of a purely additive energy type, but the physics is dramatically affected through the qualitative change of the form of the effective scalar potential in the outside "white" area (see i.e. fig. 1(b) of the first of ref.[14]), this form depending on the combination of $d_{1}$ and the sign of $k_{x}$ (see below). 
Indeed, it turns out that $V_{\text {eff }}(y)=\frac{\left(\hbar k_{x}-\frac{q}{c} A_{x}(y)\right)^{2}}{2 m}$, and in the white area $A_{x}(y)$ is a constant $-d_{1} B$, whose value is $d_{1}$-dependent, and it is matched with the form of $V_{\text {eff }}$ as this comes from inside the field at the interface; inside the field we have $V_{\text {eff }}(y)=\frac{1}{2} m \omega_{c}^{2}\left(y-y_{0}\right)^{2}$ with $y_{0}=-k_{x} \frac{\hbar c}{q B}$, and it is clear that if $d_{1}$ is not an integral multiple of $y_{0}$, then we have nontrivial consequences on the form of the potential (and therefore of the solutions) outside (whereas if $d_{1}=N y_{0}$, with $N$ integer, then $\Phi_{\text {dark }}$ is quantized and there is no new effect). In fact, the energy spectrum comes out as $\epsilon_{n_{x}, n_{y}}=\frac{\hbar^{2}}{2 m} \frac{\pi^{2} n_{y}^{2}}{d^{2}}+\frac{\hbar^{2}}{2 m}\left(\frac{2 \pi}{L}\right)^{2}\left(n_{x}-\nu\right)^{2}$, with $\nu=\frac{q d B}{h c / L}=\frac{\Phi_{\text {dark }}}{\Phi_{0}}$, and if $\nu$ is not an integer the spectrum is not equivalent to the $\nu=0$ case.

In the case of Dirac materials, by using the Dirac Hamiltonian $H=v_{f} \sigma \cdot \Pi$ (with $\boldsymbol{\Pi}=\mathbf{p}-\frac{q}{c} \mathbf{A}$ the kinematic momentum) and with ansatz $\Psi_{i}(x, y) \sim \Psi_{i}(y) e^{i k_{x} x}$ (with $i=1,2$ denoting the components of a spinor) it turns out that for the white area we have to solve a system of Schrödinger-like equations, namely $\left(-\hbar^{2} \frac{\partial^{2}}{\partial y^{2}}+\left(\hbar k_{x}-\frac{q}{c} A_{x}(y)\right)^{2}\right) \Psi_{1,2}(x, y)=$ $\frac{E^{2}}{v_{f}^{2}} \Psi_{1,2}(x, y)$, and we clearly see a similar effect as in the nonrelativistic system (the detailed solution will be given elsewhere). In case that the dark strip has no integrally-quantized flux, the solution is again not equivalent to the case $\nu=0$, especially so for a disk-geometry (with accessible $B$ ). Once again, at the bottom of this is phase-physics (and the phase-mismatch around the cylinder (or around the center in the disk) when $\Phi_{\text {dark }}$ is not quantized) [details to be published[33]]. And if we follow this method of effective scalar potential for our original striped system with the magnetic region being again inaccessible, then it turns out (in a quite different manner from what we did in the main text) that the energy spectrum in the white area is identical to eq.(1) of the text, with $f=\nu=\frac{q d_{1} B}{c h / L}$ which is $\Phi_{d a r k} / \Phi_{0}$, in agreement with our gauge transformation mapping technique. Hence the use of the effective scalar potential method - and the solution based on matching conditions in a direction transverse to the interface - seems to lead to the same results as those of a phase-mismatch analysis parallel to the interface.

In a similar vein, systems such as Zygelman's recent work 34] are also expected to be affected - if we impose periodic boundary conditions parallel to the strip - whenever the flux of the strip is not quantized (and it is easier to see this if we take the strip to be a delta function). A detailed solution will be given elsewhere[33] with the direct use of the concept of pseudomomentum (and how it is affected across the interface from inside to outside the field) and also of a new concept of pseudo-angular-momentum[35] for the corresponding 
problem in a disk geometry. However, note again that the focus of the present work is not on fields sensed by the particles, but in "forbidden" fields, because it is these cases that may make the effect of nearby fields more striking (or physically unexpected).

\section{E. Generalizing Laughlin's argument}

The folding procedure of our dark-and-white system into a cylinder, used in the main text, actually generalizes Laughlin's gauge argument on a cylinder[3], where, however, the presence of the crucial $B_{z}$ is, to our knowledge, rarely discussed. In the standard Laughlin's argument, with a radial $B$ being everywhere on the cylinder's curved cylindrical surface, one can actually understand the well-known translational symmetry breaking[36] - where the equilibrium positions of the standard Landau wavefunctions $\left(y_{0}=k_{x} l^{2}\right.$ in planar language, with $l=\sqrt{\hbar c / e B}$ the magnetic length) become priviledged[36] - by the special consideration of this additional $B_{z}$ created due to folding: as we saw, the AB flux enclosed by a horizontal circle (lying on the cylindrical surface) around the axis depends on the "height" $z$ (due to the presence of the $B_{z}=-\frac{z B}{\varrho}$ ), so that, if we want immediate wavefunction singlevaluedness around the cylinder, we indeed need special z's so that the enclosed AB flux (at that height) is quantized (in integral multiples of $\Phi_{0}$ ). It is straightforward to see that this requirement gives immediately the priviledged $z_{0}$ 's (or equivalently the above equilibrium positions $y_{0}$ 's for the standard flat Landau problem in the Landau gauge). But in our generalized system, with the area of interest (where the particle resides) being only a white strip on the cylindrical surface (with no field $B$ inside it), one finds that there are nontrivial consequences (due to remote field influence) on this white area, whenever the outside magnetic flux is not quantized. This we saw with inaccessible fields, but it seems to also occur for accessible ones as well (see Appendix D). In such case of non-quantized $\Phi_{\text {dark }}$, the wavefunction single-valuedness (or periodic boundary conditions along $x$ ) in the white area is not automatically satisfied, and it is its enforcement that leads to a modification of physical properties, hence to the remote influence of the adjacent magnetic field that we saw. A plausible question would then be: is there a "remote (or proximity) influence of the IQHE type" that might affect the particle, although this resides outside the field $B$ (hence, equivalently, a quantum Hall effect in zero-field)? There is a great deal that can be said on this, especially in cases that $B$ is accessible to the particle - i.e. in relation 
to "magnetic edge states" in the interface [37], snake states[38] etc. to be discussed in a more focused paper (the main conclusion for now being that we must have nontrivial dissipationless edge currents in the interface that, in any case, are expected, as the persistent currents associated with the hidden $\mathrm{AB}$ effect, being therefore proportional to $\left.\partial \epsilon / \partial y_{0}\right)$; but even without details, we will point out as certainly true that one can generate (or simulate) IQHE conditions on our system (always a "white area", with no B) with a pulsed outside electric field - rather than the static field case discussed in the main text and in Appendix A - which, due to its time-dependence, can induce IQHE type of effects inside our field-free system (a case involving remote electric fluxes in spacetime). An even simpler way is the main text's original example of a magnetic field $B$ in the dark area, which however is not static but slowly (adiabatically) changing with time, or, alternatively, a fixed $B$ while our origin $y_{0}$ is being displaced slowly (and transversely to the interface) between two values that correspond to a change of flux in the dark area equal to $\Phi_{0}$ (this would then define a "cycle"). This way one can achieve charge pumping (with slow variation of $B$ or of $y_{0}$ or proper combination of both) as in the case of Laughlin's cylinder[3] (replacing the much harder to build externally applied varying enclosed AB flux). After a cycle, there must be an integer number of electrons transported from one side of the system to the other (along the $y$-direction), a well-known topological quantum effect due to Thouless[39]. Or one can use

other more sophisticated types of procedures based on nonlocal terms in refs [7, 8] involving general $t$-dependent electric fields and electric scalar potentials. Summarizing, it seems that, in a number of different ways, one can induce conditions of, at least, topological (quantized) pumping of some quantity, resulting from manipulations from outside of our system, and, in fact, in ways that are expected to respect relativistic causality, as noted in the main text (and clarified in Appendix B).

\section{F. How to measure the nonlocality in a strictly planar system}

First, for a cylindrical arrangement, we have seen in the main text that the special vanishing- $B_{z}$ point $\left(z_{0}\right)$ is unique and identifiable, and survives in the $R \rightarrow \infty$ limit, so that the remote influences that are the focus of the present work must survive even after the system becomes flat; and although in the completely planar system $B_{z}$ vanishes everywhere, we have already identified the absolute reference point $z_{0}$ (or $y_{0}$ ) before the limit (as the 
unique point of vanishing $B_{z}$ that existed in the companion $3 \mathrm{D}$ system). Hence, by using this $y_{0}$, we can achieve (or measure) all the types of proximity effects discussed in the main text in the same way (namely, by placing our white area in a properly displaced manner with respect to this $\left.y_{0}\right)$.

However, for strictly planar system, when we have no knowledge of the $B_{z}$-vanishing point of a corresponding 3D companion, we argued in the main text that we have two options to consider, and here we focus on the first: If we have a large-width $\left(d_{1}\right)$ magnetic area, it is quite possible that, generically, this would behave as if it were produced by a corresponding long cylinder (in the usual theoretical limit $R \rightarrow \infty$ ) with its special vanishing- $B_{z}$ point $\left(z_{0}\right)$ being in the middle of its finite length (see again fig.3 of ref.[10] for such a system); this is for symmetry reasons and due to the fact that all expressions of the fields used here (and in fact in the whole literature) are actually exact only in the case of infinite cylinders - the middle of a long cylinder being therefore slightly preferred (as being the point that is more distant from both cylinder-ends, and also because, due to its symmetrical placement, it is a better representative of the infinite-cylinder theory). If this turns out to be correct, then this suggests an obvious experimental way on how to place our "white" area: $y_{0}$ can be taken to be in the middle of the width of the flat dark area, and then our white system must be placed as described in the main text. (Note that in this case it will be the half of the total outside flux that will remotely influence our white area, and one has interesting possibilities to study (even in case that the total outside flux is $\Phi_{0}$ ), if e.g. the spin of the electron is included). In fact, a slightly better experimental suggestion would be to have two systems of interest ("white areas", i.e. they could be identical graphene samples), separated by the above (inaccessible) wide magnetic region, and then make measurements (i.e. of persistent current) in one system or the other; the point is that, no matter where $y_{0}$ is located, at least one of the two systems must be affected by proximity (if i.e. it happens that $y_{0}$ is at the edge of one area, giving no effect on this system, then the same $y_{0}$ is necessarily displaced with respect to the 2 nd system; so proximity influence on the 2 nd system is guaranteed, if the intermediate flux is not quantized, and we can measure nontrivial effects in this 2nd system - and it is interesting to note that, if $y_{0}$ is indeed in the middle of the magnetic region, as we hoped earlier, then now, in the present setup, both systems will be affected equally). If all this does not work (meaning that there is no memory of a unique $y_{0}$, a remnant of the theoretical limit), then the lack of knowledge of a 3D companion is indeed complete, 
or equivalently this gives rise to the earlier discussed ambiguity. In such case, as noted in the main text, our criterion of proper behavior must be imposed (see the Appendices that follow).

\section{G. Dirac monopoles}

We are here interested in cases where (effective) magnetic monopoles are present. Note that, already in the case of the "Laughlin cylinder" of the main text - with the usual in the literature practice of not any mention of the extra $B_{z}$ that originates from folding of the original flat system - it is seen that the radial $B$ in 3D space must be a result of a linear magnetic monopole distribution (along the $z$-axis) - since a purely radial field violates the $\nabla \cdot \mathbf{B}=0$ law (as there is a nonzero net flux outwards and, therefore, magnetic monopoles must be invoked to justify it). And starting with an additionally placed extra narrow $(d \sim 0)$ white strip (with no field) that goes around the axis on the cylindrical surface, and imposing our criterion of decency (elimination of the gauge ambiguity) in the limit $d \rightarrow 0$ one obtains the well-known quantization of the $B$-flux in the dark area, and from this it comes out that the monopole charge must also be quantized. It is however easier to see this with a similar argument in Dirac's spherical geometry, with a single magnetic monopole at the center of a sphere[2]; enforcement then of our criterion on a small white circular section on the spherical surface around, say, the north pole, and in the limit that this section shrinks to zero (to the north pole), gives that $\Phi_{\text {sphere }}=N \Phi_{0}, N$ integer, which in turn leads to the well-known Dirac's quantization condition for the magnetic charge density $\rho_{m}$, namely $\rho_{m}=e_{m} \delta(\mathbf{r})=\frac{N h c}{4 \pi e} \delta(\mathbf{r})$, consistent with the quantization of $\Phi_{\text {sphere }}$ (to check it, recall that the radial field created by the monopole is $\mathbf{B}=\frac{e_{m}}{4 \pi r^{2}} \hat{e}_{r}$ ). [The small white section, with no field, can be achieved through the use of 2 identical spheres that are tangential to each other at a point (which will become the above north pole), with equal magnetic charges on each center - since at the tangential point the two separate fields are opposite, and they will cancel out to yield a zero total field as required for the argument; although one must be even more careful for the shake of rigor [i.e. it actually turns out that we need 3 spheres, because apart from the point of observation $\mathbf{r}$, we also want the initial point of integration $\mathbf{r}_{0}$ (that shows up in the expression of nonlocal terms) to lie outside $\mathbf{B}$, so that we actually need to consider 3 tangential spheres of equal radius with their centers lying on the $z$-axis, and with 
each one having a magnetic monopole at its center (of equal magnetic charge each); $\mathbf{r}$ will then finally be at the north pole and $\mathbf{r}_{0}$ at the south pole of the middle sphere (with both points being at zero total field, because of the cancellations), for which middle sphere we can then apply our above argument.]]

We see therefore that, by formally enforcing the elimination of this gauge ambiguity in a closed system, the nonlocal term can indeed play the role of a probe of (or a detector of) quantization of macroscopic quantities (although, it should be noted, we are merely at the level of wavefunction phases). A plausible question then is: can such a type of argumentation be followed to other more complicated cases? We answer positively and we give below, in Appendix H, some considerably more sophisticated examples, by considering topologically nontrivial systems, which - as has been shown recently[40, 41] - seem to need axion electrodynamics to describe their magnetoelectric response properties.

\section{H. Axions}

Let us first recall axion electrodynamics (but with inclusion of magnetic monopole terms, since they will be useful in our discussion of the Witten effect[5] further below). Axion electrodynamics can be described by the augmented Maxwell's equations [4]

$$
\begin{aligned}
& \nabla \cdot \mathbf{E}=4 \pi\left(\rho+\rho_{\theta}\right) \\
& \nabla \cdot \mathbf{B}=4 \pi \rho_{m} \\
& \nabla \times \mathbf{E}=-\frac{1}{c} \frac{\partial \mathbf{B}}{\partial t}-\frac{4 \pi}{c} \mathbf{J}_{m} \\
& \nabla \times \mathbf{B}=\frac{1}{c} \frac{\partial \mathbf{E}}{\partial t}+\frac{4 \pi}{c}\left(\mathbf{J}+\mathbf{J}_{\theta}\right)
\end{aligned}
$$

where the extra axionic charge and current densities are defined by $\left(\alpha=e^{2} / \hbar c\right.$ is the fine structure constant)

$$
\begin{aligned}
& \rho_{\theta}=-\frac{\alpha}{(2 \pi)^{2}} \nabla \cdot(\theta \mathbf{B})=-\frac{\alpha}{(2 \pi)^{2}}(\nabla \theta \cdot \mathbf{B}+\theta \nabla \cdot \mathbf{B}) \\
& \mathbf{J}_{\theta}=\frac{c \alpha}{(2 \pi)^{2}} \nabla \times(\theta \mathbf{E})+\frac{\alpha}{(2 \pi)^{2}} \frac{\partial}{\partial t}(\theta \mathbf{B})=\frac{c \alpha}{(2 \pi)^{2}}(\nabla \theta \times \mathbf{E}+\theta \nabla \times \mathbf{E})+\frac{\alpha}{(2 \pi)^{2}}\left(\theta \frac{\partial \mathbf{B}}{\partial t}+\mathbf{B} \frac{\partial \theta}{\partial t}\right) .
\end{aligned}
$$

In particular, note the continuity equation for the $\theta$-terms, namely $\nabla \cdot \mathbf{J}_{\theta}+\frac{\partial \rho_{\theta}}{\partial t}=0$ (this basically reflecting the conserved "Witten electric current" - see mention of the Witten effect further below).

As is well-known, the above originate from an extra term in the electromagnetic Lagrangian density, that is of the form $£_{\text {axion }}=\theta\left(\frac{e^{2}}{2 \pi h c}\right) \mathbf{E} \cdot \mathbf{B}=\theta \frac{\alpha}{(2 \pi)^{2}} \mathbf{E} \cdot \mathbf{B}$, which is periodic 
with respect to $\theta$ with period $2 \pi$ (and if $\theta$ is static, it only takes values 0 or $\pi$ for t-reversalsymmetric systems - 0 being the value for conventional, and $\pi$ for topologically nontrivial systems). The proof of this periodicity that has been recently given by Vazifeh \& Franz[17] in the absence of magnetic monopoles can actually be given directly by our natural criterion, by actually justifying better the separate quantization of certain fluxes that does not seem to be justified in ref.[17]. For the example of [17] with $\mathbf{B}=B_{z} \hat{e}_{z}$ and $\mathbf{E}=E_{z} \hat{e}_{z}$, we see that our nonlocal fluxes appear naturally after integration (over spatial and time variables) of $£_{\text {axion }}$ in order to obtain the axionic action $S_{\text {axion }}$, namely $S_{\text {axion }} / \hbar=\frac{\theta}{\Phi^{2}} \int B_{z} d x d y \int E_{z} c d t d z$. And although the separate quantization of the fluxes in eq.(16) of ref.[17] does not seem to result from any basic principle, it is justifiable by our quantization of nonlocal terms (the one that appears above with the $B_{z}$, a usual magnetic flux, and the one with $E_{z}$, a spacetime electric flux). So the separate quantization gives $n_{1} \Phi_{0}$ for the magnetic flux and $n_{2} \Phi_{0}$ for the electric flux $\left(n_{1}, n_{2}\right.$ integers $)$, so that finally $S_{\text {axion }} / \hbar=N \theta\left(N=n_{1} n_{2}\right)$ as we were seeking to prove. In a sense, the above separate quantization of magnetic and electric fluxes proves that the axionic action (which is a 2nd Chern number for this Abelian gauge theory) turns out to be a product of two 1st Chern numbers, whose quantization comes out directly by imposing our criterion of proper behavior (i.e. enforcing the elimination of gauge ambiguity in the planes $(x y)$ and $(t z))$, and without further topological considerations.

Returning to the above generalized Maxwell's equations, note that $\int \nabla \cdot \mathbf{J}_{\theta} d t=-\rho_{\theta}=$ $\frac{\alpha}{(2 \pi)^{2}} \nabla \cdot(\theta \mathbf{B})$, so that its volume integral in a spatial region will give a flux of $\theta \mathbf{B}$ through the surface boundary. And because $\theta$ just suffers a jump by $\pi$ at the surface (if this is the interface between an axionic medium and a conventional one, i.e. the vacuum) there remains just the magnetic flux through the surface; hence our criterion of its flux quantization leads naturally to the quantization of $\mathbf{J}_{\theta}$. However, as has been noted in the past as an observation, $\mathbf{J}_{\theta}$ can describe the Hall current, $\mathbf{J}_{\theta}=\mathbf{J}_{\text {Hall }}$ (see below for a new and clear proof), hence the above conclusion on $\mathbf{J}_{\theta}$ leads to an immediate understanding of the quantization of the Hall response. But what type of quantization? (It will turn out in the following that there are two types, integral and "half-integral").

Let us first consider a conventional 2D Quantum Hall $(\mathrm{QH})$ sheet (i.e. with $\sigma_{H}=-\nu e^{2} / h$, with $\nu$ an integer (the filling factor in a Landau level picture - or, more generally, the 1st Chern number in the Brillouin zone of a periodic system)) and let us fold it along the $x$ - 
direction into a cylinder. Applying an electric field $E$ on the surface, with $E$ parallel to the cylinder axis, we obtain a transverse Hall current $I$ (hence in the azimuthal direction) of density $J=\sigma_{H} E$, where $J=I / d$ (with $d$ the height of the cylinder). We have therefore a total magnetic moment $M_{t o t}$ induced that is parallel to the $z$-axis and has a value $M_{t o t}=-\frac{I S}{c}$ (this comes out if we view $I$ as related to the magnetization current through $\mathbf{J}=\mathbf{J}_{\text {magn }}=$ $c \nabla \times \mathbf{M}) \Rightarrow \tilde{\mathbf{n}} \times\left(\mathbf{M}_{2}-\mathbf{M}_{1}\right)=\frac{1}{c} \mathbf{K}_{\text {magn }}$, with $\mathbf{K}_{\text {magn }}$ the surface current per transverse length, and with the cross section $S=\pi R^{2}$, so that $M_{t o t}=-\sigma_{H} \frac{S}{c} d E=-\nu \frac{e^{2}}{h} \frac{V}{c} E$ (where we wrote $S d=V$ the volume of the cylinder). We have therefore an induced (by the electric field) magnetization $M=\frac{M_{t o t}}{V}=-\nu \frac{e^{2}}{h c} E$. Now, it is well-known (and it results from variation of the above $S_{\text {axion }}$ ) that axionic physics leads to magnetoelectric effects such that an electric field $\mathbf{E}$ induces a parallel magnetization $\mathbf{M}=\frac{\alpha}{4 \pi} \frac{\theta}{\pi} \mathbf{E}$, whereas a magnetic field $\mathbf{B}$ induces a parallel polarization $\mathbf{P}=\frac{\alpha}{4 \pi} \frac{\theta}{\pi} \mathbf{B}$. The above picture of the IQHE already leads to (recall that $\left.\alpha=e^{2} / \hbar c=2 \pi \frac{e^{2}}{h c}\right)$ a magnetization $M=-\nu \frac{e^{2}}{h c} E=-\nu \frac{\alpha}{2 \pi} E$ which corresponds to the above general result with $\theta=2 \pi \nu$. (This probably demonstrates in a sense the conventional character of the IQHE). We can then actually show that this correspondence $(\theta=2 \pi \nu)$ is also valid for the polarization induced by a magnetic field. Indeed, by applying a $\mathbf{B}$ in all space parallel to the cylinder axis we obtain as a response an electric charge density, say $n_{A} e$ $\left(n_{A}\right.$ being the areal number density of charge carriers) induced on the ceiling of the cylinder - and one of the same magnitude induced on the floor, but with opposite sign (hence we do indeed have a dipole electric moment and therefore a polarization induced, parallel to the axis) - a result that comes from the well-known Streda formula [42] $\sigma_{H}=-\frac{\partial\left(e n_{A} c\right)}{\partial B}$ (see below), and the $B-n_{A}$ "locking formula" of the IQHE, namely $B=\frac{n_{A} \Phi_{0}}{\nu}$ (that connects the constant (and very robust) value of $B$ at the plateau labeled by the integer $\nu$ in an IQHE experiment), or in an even more elementary manner by the polarization charge density $\rho_{\text {pol }}=-\nabla \cdot \mathbf{P} \Rightarrow\left(\mathbf{P}_{2}-\mathbf{P}_{1}\right) \cdot \tilde{\mathbf{n}}=-\sigma_{\text {pol }}$ the surface polarization charge density induced on each considered surface. Let us check whether this polarization response satisfies the above general axionic expression, again with $\theta=2 \pi \nu$ : indeed, from Streda formula $\sigma_{H}=-\frac{\partial\left(e n_{A} c\right)}{\partial B}$ together with $n_{A}=\frac{\nu B}{\Phi_{0}}$ we get $\sigma_{H}=-\frac{\partial}{\partial B}\left(e \frac{\nu B}{\frac{h c}{e}} c\right)=-\nu \frac{e^{2}}{h}$ the correct Hall conductance, and therefore the total electric moment induced in the $z$-direction is $P_{t o t}=-e n_{A} S d=-e \frac{\nu B}{\frac{h c}{e}} V$; hence finally the polarization is $P=\frac{P_{t o t}}{V}=-\nu \frac{e^{2}}{h c} B=-\nu \frac{\alpha}{2 \pi} B$, and we see that this indeed also satisfies the above general axionic response relation, with $\theta=2 \pi \nu$.

Along similar lines one can find in the literature[43] the manner in which one can obtain 
the anomalous (half-integral) Quantum Hall Effect at the surface of a strong topological insulator, by using an argument such as the above, with $\theta=\pi$ for the topological insulator and $\theta=0$ for vacuum (hence the above spatial derivatives of $\theta$ give delta function contributions (from $\rho_{\theta}=-\frac{e^{2}}{2 \pi h} \nabla \theta(\mathbf{r}) \mathbf{n} \cdot \mathbf{B}$ and $\mathbf{J}=c \frac{e^{2}}{2 \pi h} \nabla \theta(\mathbf{r}) \times \mathbf{E}$ at the interface, as we move from one medium to the other)). Indeed, for a flat $2 \mathrm{D}$ system one obtains $\rho_{\theta}=\frac{\alpha}{4 \pi^{2}} \delta(z) B_{z}$ and $\mathbf{J}_{\theta}=-\frac{\alpha}{4 \pi^{2}} \delta(z) \hat{e}_{z} \times \mathbf{E}$ (for each surface of a topological insulator film) and it finally turns out [44] that $\sigma_{H}=\left(\theta_{1}-\theta_{2}+2 \pi n\right) \frac{e^{2}}{2 \pi h}$, leading (i.e. for $\theta_{1}=\pi$ and $\theta_{2}=0$ ) to the "half-integral" quantization for topological insulators, namely $\sigma_{H}=\frac{e^{2}}{2 h}$ modulo $\frac{e^{2}}{h}$, or simply $\sigma_{H}= \pm \frac{e^{2}}{2 h}$. And this, applied to the surface of a topological insulator cylinder (in a way similar to our above application to an IQHE system) also gives rise to a quantized magnetoelectric response $\mathbf{M}=\frac{\alpha}{4 \pi} \mathbf{E}$ and $\mathbf{P}=\frac{\alpha}{4 \pi} \mathbf{B}$, compatible with the above discussion and quite generally expected for topologically nontrivial materials (corresponding to $\theta=\pi$ ). Note that deep down, the origin of the above is essentially the Witten effect [5], which is usually presented as follows [45]: from $\nabla \cdot \mathbf{E}=4 \pi\left(\rho+\rho_{\theta}\right)$ and considering now $\theta$ to be a function of time only $\theta(t)$, and using the possible presence of magnetic monopoles through $\nabla \cdot \mathbf{B}=4 \pi \rho_{m}$, it turns out that $\int \nabla \cdot \mathbf{E} d^{3} r=4 \pi \int d^{3} r\left(\rho(\mathbf{r})-\frac{\theta}{\pi} \alpha \rho_{m}(\mathbf{r})\right)$; hence if the total charge is $Q=\int d^{3} r \rho(\mathbf{r})=-N e$, and $\theta=\pi$, and because $4 \pi \int \rho_{m} d^{3} r=n \Phi_{0}$ (the Dirac quantization, as derived earlier), we get $\int \alpha \rho_{m} d^{3} r=n e / 2$, or that effectively we have a charge $Q_{e f f}=-e\left(N-\frac{n}{2}\right)$, namely an effective charge that is generally half-integral[5]. Summarizing, we see that imposition of our criterion of proper behavior (that enforces elimination of our gauge ambiguity) leads to quantization of $\mathbf{J}_{\theta}$, which in turn leads, for conventional IQHE systems to $\sigma_{H}=$ an integral multiple of $\frac{e^{2}}{h}$, and for topological insulator surfaces that are in contact with a topologically trivial medium (i.e. the vacuum) to $\sigma_{H}=$ an odd integral multiple of $\frac{e^{2}}{2 h}$; it also leads to their quantized magnetoelectric responses, as these were discussed above.

One can actually generalize the above magnetoelectric effects to more general topologically nontrivial quantum devices, combined with the field-effect from a distance (the central result of the main article), but this is reserved for a future discussion. 


\section{Predictions on devices, Graphene and Topological Insulators}

Descriptions of possible types of measurements (related to the gauge nonlocality effect presented in this work) in conventional systems have been briefly given in the main text and in Appendix E (mostly on induction of IQHE from outside the system). It should be stressed, as a generic feature (and prediction) that, even if our "white area" is empty (i.e. single electron in empty space), we would at least expect (persistent) currents along the edge (interface between white and dark areas) - this being valid for both parabolic and Dirac electronic spectrum[46]. This was also noted in the main text (with the expectation that $J$ will be proportional to $\left.\partial \epsilon / \partial y_{0}\right)$.

Here is what one would expect on general grounds, if our white system is graphene or a topological insulator: Graphene: proximity arrangement with a B, would offer a controllable way (through changes of the outside $\mathbf{B}$ or of $y_{0}$ ) to lift the orbital degeneracy that originates from the two valleys (even without inter-valley scattering), with consequences on persistent currents (in $x$-direction) and in conductance (i.e. some shifting of peaks), analogous to the

ones of ref.[47]. In addition, giant magnetoresistance at room temperature is possible, due to the hidden AB interference[48]. Topological insulators: By way of an example, in the proximity of an HgTe quantum well one would expect to measure helical edge states, bound states and persistent currents (with Rashba spin-orbit coupling), that would generally be affected in a manner similar to the one described in ref.[49]. On all this, we plan to return with details in a future note.

\section{J. Formal analogies with other areas}

Finally, the purpose here is to examine the wider physical implications, and/or relationships with other physics areas that we see formal connections with.

1. For time-dependent fields, the time-derivative of the phase-nonlocalities noted in the present work (and expressed through the nonlocal terms of Appendix B) seems to be directly related to recent considerations of Berry and Shukla[21] on "curl forces" that are spatially confined in classical systems (while the point of observation is outside, in curl-free regions), giving simultaneously their quantum generalization (to be addressed in separate work). A "curious evocation of the $A B$ effect" is a statement mentioned twice in [21], and it will 
be shown that this is actually related to our nonlocal terms, and to the nonlocal gauge influences that generally show up in the spirit of the present work (and more concretely, to the hidden $\mathrm{AB}$ effect in some 3D companion system).

2. For a many-body system of non-interacting electrons there will be interesting transitions (upon variation of the width $d$ of the "white" area), such as the ones recently worked out in detail in 3D systems[50] (note in particular that there is a gap opening due to the nonzero values of $k_{y}$ and this will have consequences). The above is for our simple "empty" problem, but can also be worked out in a "white" graphene sample (a Dirac material, outside a nearby field, now with electron and hole bands taken into account).

3. An obvious generalization of these proximity influences to a many-body system with electron-electron interactions (but with no magnetic field inside our system) leads to another novel possibility, of potential relevance to the physics of composite fermions without the presence of a magnetic field (where the extra vector potentials induced by proximity can lead to corresponding Chern-Simons physics, in a manner similar to the one studied recently in ref.[24]).

4. Possible applicability to other systems (i.e. that involve rotations rather than magnetic field (due to the well-known formal similarity between the two physical situations) and that give rise to analogies with the $\mathrm{AB}$ effect, with i.e. water waves, pioneered by Berry [51]): such systems have been recently noted to exhibit quantization of orbits as well as nonlocality (generated by path memory) - see the very recent preprint [52], and the gauge nonlocality advanced in the present work might be directly applicable.

5. Spin-physics, through a combination of remote field influences with proper Lorentz boosts: it is well-known (see i.e. ref.[53]) that in setups such as the ones discussed in the main text (i.e. the initial example of an orthogonal strip), if one boosts to a moving frame with velocity $\mathbf{v}=c \frac{\mathbf{E} \times \mathbf{B}}{E^{2}}$, then, as a result, the moving observer perceives a magnetic moment $\mu=\frac{1}{2} q \mathbf{r} \times \mathbf{v}$ for the particle. Using then $\mathbf{B}=\frac{1}{c} \mathbf{v} \times \mathbf{E}$, it turns out that the moving observer experiences the vector potential as $\frac{q \mathbf{A}}{c}=\frac{1}{c^{2}} \mu \times \mathbf{E}$, namely a vector potential of the AharonovCasher type[29]. All the earlier quantization conclusions can be therefore transported to quantization and IQHE-type of phenomena that concern the particle's magnetic moment and spin. We shall return to this in a future article, but for here it suffices to note that nontrivial spin-physics can be studied this way, starting from purely orbital considerations. 
6. One cannot stop wondering whether the new nonlocal terms (basically responsible for the above effects (see Appendix B), viewed as generalized solutions of $\nabla \Lambda=\mathbf{A}$ on the simple-connected plane, but in problems where $\Lambda$ is not defined everywhere as a single-valued function) can have an impact on other areas of physics where we have such partial differential equations on a plane. One example of applicability that we already saw is the Berry \& Shukla problem[21] mentioned earlier in this Appendix. Another immediate candidate is the entire area of thermodynamics, filled with 1st order partial differential equations of this form, and it seems that, indeed, the nonlocal terms that appear in their solutions might have connection to issues of irreversibity and vorticity[22] that, for now, go much further than the scope of this paper.

7. Finally, and again going much beyond the scope of the present article, one cannot help noticing that there seems to be a general connection of the above with certain high-energy physics phenomena. Although the physics is very different (the dynamical variables involved also being different), there seems to be a formal relationship that might be useful (at least through analogies). Such a formal analogy is the recent work in Maxwell electrodynamics on a compact manifold, that finds a topological contribution to the Casimir force[18] that seems to be the formal analog of the persistent currents asserted here (upon variation of $\left.y_{0}\right)$ due to the proximity effect of the present work. More generally, note earlier works on $\theta$-vacuum (see i.e. ref.[19]) with $\theta$-vacuum sectors being formally analogous to our $y_{0}-$ sectors (which - if they were dynamic (i.e. if the 3D B-construction or the cylinder had their own dynamics (i.e. a vibrational one)) - they would be analogous to axions). In fact, in ref.[18] on topologically inequivalent (winding) states, where, in addition, a direct connection of $\theta$-vacua with magnetic fields is made, one can see a similar formal analogy with our simpler system; in a sense, our work points out to another "amazing example" (in the language of the authors of ref.[18]) - now in low-energy physics - where the external $B$ is now outside the system (playing the role of the $\theta$-parameter)). Similarly, one can see some possible formal analogy of our $y_{0}$-ambiguity to the so-called Gribov problem (or Gribov ambiguity[20]). This will be valid, when the Gribov copies are gauge-equivalent configurations that satisfy the Landau gauge condition [54]. For such a claimed connection see in particular refs [55] and [56] where the existence of the Gribov phenomenon is related to the existence of inequivalent quantizations (which in our simpler problem means different 
$y_{0}$-sectors), and therefore Gribov copies are labeled through procedures that are formally similar to ours; and this is done for abelian gauge fields at zero temperature (as noted in ref.[57]) - although originally Gribov copies were discovered for only non-abelian gauge fields at a general finite temperature. In particular there is an issue of the Gribov problem showing up when gauge fields (like our potentials) do not vanish at infinity - and it seems that a similar issue that has gone largely unnoticed exists here as well: i.e. in the majority of literature on $\mathrm{AB}$ effects (see as an example the very recent work of Stewart[58]) there is a proof in Coulomb gauge that there is no further gauge ambiguity, the reason being the vanishing at infinity of vector potential $A$ in a standard $\mathrm{AB}$ configuration with cylindrical symmetry (in which case, $A$ goes as $1 / \varrho$ outside the enclosed flux and indeed vanishes at infinity); this however is not true if the inaccessible region is i.e. a rectangle (and it is not true for our striped geometry either), because in such orthogonal geometries (where our gauge can be used) the corresponding $A$ outside the field is a nonzero constant that can go up to infinity. Hence a basic assumption, usually made implicitly, does not hold in our case (and mathematically speaking it leads to the gauge ambiguity emphasized in the present work), and something formally similar seems to hold in high energy physics [59] for systems that exhibit the Gribov ambiguity. Needless to say, all these issues are only briefly mentioned here without justification and require closer scrutiny; however, we feel it is useful to point them out, in case that the gauge nonlocality and the associated proximity effect found in the present work, might be the low-energy analog of previously known (but mostly esoteric) technical matters in high-energy physics, contributing therefore to their demystification (and, possibly, vice versa: bringing out some esoteric and sophisticated behavioral patterns, that may be hidden in an "ordinary" solid state system in the laboratory, and with the actual possibility of experimental and practical applications). Such analogies would be useful for possibly making further progress in deeper gauge-related issues in both high- and low-energy physics, and this is why they deserve to be investigated further.

[1] Y. Aharonov \& D. Bohm, Physical Review 115, 485 (1959)

[2] P. A. M. Dirac, Proceedings of the Royal Society A133, 60 (1931) 
[3] R. B. Laughlin, Physical Review B 23, 5632 (1981)

[4] F. Wilczek, Physical Review Letters 58, 1799 (1987)

[5] E. Witten, Physics Letters B 86, 283 (1979)

[6] M. Bawin \& A. Burnel, Journal of Physics A 18, 2123 (1985)

[7] K. Moulopoulos, Journal of Physics A, 43, 354019 (2010)

[8] K. Moulopoulos, Journal of Modern Physics 21, 1250 (2011)

[9] C. Chryssomalakos et al., European Journal of Physics 25, 489 (2004)

[10] E. Westerberg \& T. H. Hansson, Physical Review B 47, 16554 (1993)

[11] M. D. Semon \& J. R. Taylor, American Journal of Physics 64, 1361 (1996)

[12] V. Jakubsky et al., arXiv:1306.2115; see also S. Roche et al., Physical Review B 62, 16092 (2000)

[13] N. Agrawal et al., International Journal of Modern Physics B 27, No. 10, 1341003 (2013)

[14] F. M. Peeters \& A. Matulis, Physical Review B 48, 15166 (1993); A. Matoulis, F. M. Peeters \& P. Vasilopoulos, Physical Review Letters 72, 1518 (1994)

[15] M. R. Masir, P. Vasilopoulos \& F. M. Peeters, Journal of Physics: Condensed Matter 22, $465302(2010)$

[16] Z. Wu, F. M. Peeters \& K. Chang, Physical Review B 82, 115211 (2010)

[17] M. M. Vazifeh \& M. Franz, Physical Review B 82, 233103 (2010))

[18] C. Cao, M. van Caspel and A. R. Zhitnitsky, Physical Review D 87, 105012 (2013)

[19] M. Asorey, J. G. Esteve \& A. F. Pacheco, Physical Review D 27, 1852 (1983)

[20] V. N. Gribov, Nuclear Physics B 139, 1 (1978)

[21] M. V. Berry \& P. Shukla, Journal of Physics A 45, 305201 (2012)

[22] See i.e. V. E. Shapiro, arXiv:1305.5108; also unpublished work of R. M. Kiehn on the web

[23] M. Kohmoto, Annals of Physics, 160, 343 (1985)

[24] V. M. Gvozdikov, Physical Review B 82, 235110 (2010)

[25] M. V. Berry, Proceedings of the Royal Society of London A 392, 45 (1984)

[26] K. Moulopoulos \& M. Constantinou, Physical Review B 70, 235327 (2004); 76, 039902 (2007)

[27] K. Kyriakou, K. Moulopoulos, A. V. Ghazaryan et al., Journal of Physics A 43, 354018 (2010)

[28] D. R. Hofstadter, Physical Review B 14, 2239 (1976)

[29] Y. Aharonov \& A. Casher, Physical Review Letters 53, 319 (1984)

[30] K. Kyriakou \& K. Moulopoulos, to be submitted 
[31] K. Moulopoulos, in preparation

[32] Y. Aharonov \& D. Rohrlich, "Quantum Paradoxes", J. Wiley, Berlin (2005); J. Oppenheim \& S. Wehner, Science 320, 1072 (2010)

[33] G. Konstantinou \& K. Moulopoulos, in preparation

[34] B. Zygelman, Physical Review A 86, 042704 (2012), Appendix B

[35] K. Kyriakou \& K. Moulopoulos, in preparation

[36] S. Jansen, E. H. Lieb \& R. Seiler, Communications in Mathematical Physics 285, 503 (2009)

[37] P. D. Hislop \& E. Soccorsi, arXiv:1307.5968

[38] J. E. Muller, Physical Review Letters 68, 385 (1992)

[39] D. J. Thouless, Physical Review B 27, 6083 (1983)

[40] X. Qi, T. L. Hughes \& S. -C. Zhang, Physical Review B 78, 195424 (2008)

[41] A. M. Essin, J. E. Moore \& D. Vanderbilt, Physical Review Letters 102, 146805 (2009)

[42] P. Streda, Journal of Physics C 15, L717 (1982)

[43] S. Ryu, J. E. Moore \& A. W. W. Ludwig, Physical Review B 85, 045104 (2012)

[44] M. Sitte et al., Physical Review Letters 108, 126807 (2012)

[45] G. Rosenberg \& M. Franz, Physical Review B 82, 035105 (2010)

[46] D. Sticlet et al., arXiv:1307.6964

[47] P. Recher et al., Physical Review B 76, 235404 (2007)

[48] V. H. Nguyen et al., arXiv:1303.4946

[49] P. Michetti \& P. Recher, Physical Review B 83, 125420 (2011)

[50] G. Konstantinou \& K. Moulopoulos, European Physical Journal B 86, 326 (2013)

[51] M. V. Berry et al., European Journal of Physics 1, 154 (1980)

[52] E. Fort et al., arXiv:1307.6051

[53] L. L. Xu et al., Integrated Ferroelectrics 131, 36 (2011)

[54] P. M. Lavrov \& A. A. Reshetnyak, arXiv:1210.5651

[55] E. Langmann \& G. W. Semenoff (1993), Physics Letters B 303, 303 (1993)

[56] J. E. Hetrick (1993), Nuclear Physics Proceedings Supplement 30, 228 (1993)

[57] R. Parthasarathy, Letters in Mathematical Physics 15, 179 (1988)

[58] A. M. Stewart, Canadian Journal of Physics 91, 373 (2013)

[59] J. R. Ellis, Journal of Physics A 23, 65 (1990) 